\title{
RESPUESTA ESTATAL POR PRESIONES EXTERNAS: LOS DETERMINANTES DEL FORTALECIMIENTO ESTATAL Frente Al BOOM DEL ORO EN El Perú (2004-2015)*
}

\author{
State Response by External Pressures: The Determinants of the State's \\ strengthening to face the Illegal Gold Mining Boom in Peru (2004-2015)
}

\section{EDUARDO DARGENT}

Pontificia Universidad Católica del Perú

\section{MADAI URTEAGA}

Pontificia Universidad Católica del Perú

\begin{abstract}
RESUMEN
La minería informal e ilegal se ha configurado como un poderoso desafío para el Estado peruano en el contexto del boom de recursos que vive el país. Este artículo documenta y explica la respuesta del Estado a este fenómeno. Luego de una primera etapa de inacción (2004-2008), el Estado ensaya una respuesta desarticulada (2008-2011) para finalmente establecer una más coherente y organizada (2011-2015). Aunque todavía insuficientes, estos cambios han dado lugar a un mayor fortalecimiento de las áreas del Estado a cargo de enfrentar el fenómeno. Proponemos que esta evolución de la respuesta institucional se explica por factores externos al Estado (presiones internacionales y demandas domésticas) antes que por procesos producidos en su interior.
\end{abstract}

Palabras clave: capacidad del Estado, oro, economías ilegales, Perú, boom de commodities

\begin{abstract}
In the context of the current commodity boom, informal and illegal mining have become acute challenges to the Peruvian state. This article documents and explains the state's response to these challenges. After a stage of inaction (2004-2008), the Peruvian state initially reacted with a disarticulated response (2008-2011) before mounting a more coherent and organized response (2011-2015). Although the state's response to the challenge has been limited, it is has nevertheless resulted in an observable increase in state capacity. We propose that this phenomenon is better explained by factors external to the state (international pressures and domestic demands) rather than by processes triggered from within.
\end{abstract}

Key words: state capacity, gold, illegal economies, Peru, commodity boom.

Este trabajo fue realizado en el marco del proyecto de investigación "Capacidad de respuesta del Estado en el Perú: Crisis, desafíos y entorno cambiante" desarrollado por el Grupo de Investigación sobre Capacidad del Estado Peruano (GICEP) y financiado por la PUCP. Agradecemos a los integrantes del grupo, a Maritza Paredes y Alisha Holland por sus valiosos comentarios a versiones anteriores de este trabajo, así como a los dos revisores anónimos por sus contribuciones a la mejora del texto. 


\section{INTRODUCCIÓN}

Desde el año 2004 el abrupto aumento del precio de los minerales tuvo considerables impactos sociales, económicos y políticos en el Perú (Damonte 2013; Orihuela 2014). El oro es uno de los metales que más ha subido su cotización en el mercado internacional. En la última década el precio del mineral se incrementó hasta en un 360\% en comparación con el 2004 y si bien en los dos últimos dos años descendió ligeramente, aún se encuentra muy por encima del que tenía al inicio del boom (ver gráfico 1). La extracción de oro es relativamente sencilla y altamente rentable. Por ello, a la vez que su precio se incrementó, lo hicieron también la minería ilegal e informal en distintos departamentos del Perú así como actividades ilegales conexas. Así pues, el boom del oro se ha configurado como un enorme reto para el Estado peruano.

De acuerdo con la ley peruana, la diferencia entre la minería informal y la minería ilegal es que la primera posee potencial de formalización; es decir, puede convertirse en una actividad permitida con la autorización del Estado. En contraste, la minería ilegal opera en zonas prohibidas para la explotación de recursos naturales y, por tanto, debe ser erradicada (Defensoría del Pueblo 2014: 165). En ambos casos estamos ante conductas que vulneran normas estatales con graves consecuencias ambientales y sociales.

Gráfico 1: Producción informal y cotización del oro

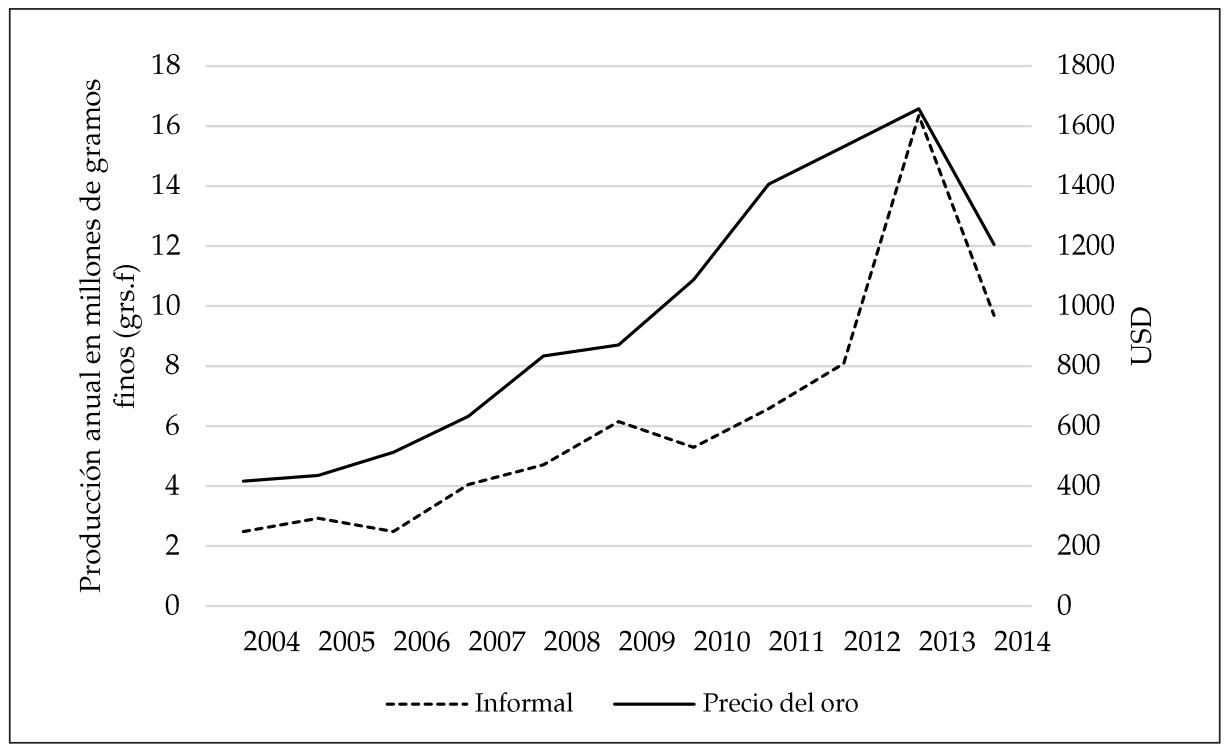

Fuente: MINEM. Elaboración propia.

Partiendo de la literatura sobre capacidad estatal y los determinantes políticos de la acción del Estado, este artículo evalúa la respuesta del Estado peruano 
al desafío del oro ilegal y explica qué factores la determinaron. Si bien esta respuesta sigue siendo en general limitada frente al fenómeno, ${ }^{2}$ es posible observar un gradual fortalecimiento de ciertas áreas del Estado que permite una acción más comprehensiva y coordinada. A través de la revisión de fuentes secundarias (reportes estatales, archivos de noticias, informes de la sociedad civil, datos agregados) y entrevistas a actores estatales y de la sociedad civil del nivel nacional y subnacional en el departamento de Puno, mostramos que la respuesta estatal ha evolucionado en tres etapas claramente distinguibles: (i) inacción (2004-2008), (ii) respuesta desarticulada (2008-2011), (iii) respuesta organizada (2011-2015). Las etapas dos y tres evidencian cambios muy claros respecto a la primera que implicaron la instauración de nuevas reglas, creación de agencias y oficinas para controlar el fenómeno, inversión de mayores recursos financieros y aumento de personal. Como resultado, aquellas partes del Estado a cargo de responder al desafío han aumentado su capacidad.

Proponemos que esta respuesta del Estado se explica por factores externos a este más que por procesos o decisiones internas. Un Estado atravesado por la debilidad a nivel central y regional, y atrapado en el marco de una descentralización iniciada apenas un año antes del boom, no fue la principal fuente de respuesta frente al desafío del oro. Alternativamente, se adaptó de forma reactiva frente a presiones internacionales, tratados comerciales que obligaron al Estado a crear instituciones ambientales como el Ministerio del Ambiente (MINAM), y la demanda de grupos ambientalistas trasnacionales, y domésticas, tales como el reclamo de los sectores mineros formales. Entonces, cuando el Estado reaccionó, como cuando el MINAM intentó tomar la dirección del proceso (2009) o cuando se creó la Oficina del Alto Comisionado para la Minería Ilegal (2014), lo hizo empujado por estas presiones.

Como discutimos en la conclusión, esta forma de fortalecimiento reactivo arroja dudas tanto sobre la sostenibilidad de la respuesta estatal frente al desafío, así como sobre la capacidad generada en el proceso. ¿Las prioridades del Estado se han enfocado en la problemática general o más bien han sido determinadas por la agenda de las demandas externas? ¿Las oficinas actuales han alcanzado suficiente capacidad como para mantener la relevancia y urgencia del tema? ¿O ante la ausencia o debilitamiento de presiones externas al Estado estas áreas perderán su relevancia?

Es importante aclarar que no se propone que las demandas externas hayan producido una respuesta necesariamente efectiva del Estado, pues como se mencionó en general la respuesta es todavía limitada. La "efectividad", por lo demás, es un estándar complejo de evaluar en casos como este; hasta los Estados más fuertes tienen problemas para enfrentar a retadores que emergen o se fortalecen durante un boom de recursos. Lo que nos interesa mostrar es 
que estas demandas externas fueron las que hicieron reaccionar al Estado y lo llevaron a fortalecerse en términos de incremento de recursos materiales y burocráticos. En esa línea, este estudio de caso busca contribuir a la creciente literatura sobre los determinantes políticos de la acción del Estado señalando otro mecanismo que lo impulsa a ejercer su autoridad en el territorio. Además de la intencionalidad política y los vínculos con la sociedad, ${ }^{3}$ las presiones externas, ya sean internacionales y/o domésticas, dan lugar a que incluso un Estado débil con pasivos decida responder a un fenómeno que alternativamente habría preferido ignorar ante la ausencia de las mismas.

El orden del artículo es el siguiente. Tras una breve introducción teórica sobre los desafíos al Estado, capacidad estatal y los determinantes políticos de la respuesta estatal, discutimos en la segunda sección las implicancias del desafío del oro ilegal. Luego documentamos las tres etapas de la respuesta estatal. En la sección principal del artículo mostramos cómo esta respuesta estuvo determinada por presiones externas y no por procesos al interior del Estado y señalamos cómo estas presiones dieron forma a la respuesta del Estado. Concluimos con algunas lecciones del caso en cuanto a la construcción de capacidad Estatal y sugiriendo una posible agenda de investigación comparada.

\section{BOOM DE RECURSOS, DESAFÍOS, RETADORES Y CAPACIDAD ESTATAL}

Los booms de recursos son periodos de excepcional abundancia de rentas para el Estado provenientes de actividades económicas primarias mineras y / o agrícolas. Si bien estos periodos de bonanza dan oportunidades a los Estados, dotándolos de recursos económicos, también incrementan viejos problemas o crean nuevos (Saylor 2014; Kurtz 2013; Paredes 2013). Una forma en que los booms de recursos pueden ser problemáticos es cuando alimentan el surgimiento y fortalecimiento de desafíos y retadores que amenazan la autoridad del Estado. De acuerdo con Eaton (2012), los desafíos al Estado son situaciones que impactan en el aparato estatal cuestionando su autoridad y demandan una respuesta del mismo para contrarrestarla. Estos desafíos suelen tener como protagonistas a retadores al Estado, los cuales son actores, violentos o no violentos, que disputan, objetan o resisten la autoridad del mismo para implementar sus decisiones y políticas (Eaton 2012: 648-649). Ejemplos de desafíos contra el Estado que pueden nutrirse directa o indirectamente del boom son el narcotráfico, las guerrillas, el aumento de grupos extorsivos, o, en el caso que nos ocupa, la minería informal e ilegal.

¿Cómo reacciona un Estado frente a estos desafíos? ¿Qué determina la efectividad y calidad de esa respuesta? La literatura sobre capacidad estatal resalta que los Estados fuertes tienen más posibilidad de imponer su autoridad 
frente a los desafíos que enfrenten mientras que los débiles probablemente fracasarán. Así, el Estado usa sus recursos materiales y burocráticos para hacer respetar sus reglas y brindar una respuesta efectiva. Giraudy (2012), considera a un Estado capaz cuando posee una burocracia profesional, mantiene cierta distancia e independencia de actores sociales dominantes (autonomía) y tiene presencia efectiva en el territorio. La idea central es que las agencias del Estado, gracias a contar con estas características, pueden prever y responder los desafíos que enfrentan, siendo capaces de ajustar las políticas públicas y fortalecer sus agencias cuando estas fallan (Stein y Tomassi 2007: 7). En suma, dado que el Estado es capaz, el motor de una respuesta efectiva se gesta en su interior. ${ }^{4}$

Al contrario, se espera que los Estados débiles no reaccionen en forma adecuada frente a un desafío. Si bien todo Estado, fuerte o débil, enfrenta un reto mayúsculo al lidiar con retadores nutridos por un boom de recursos como el actual, los Estados débiles tendrán menos posibilidad de adaptarse y responder. Con burocracias poco profesionales, frecuentemente penetradas por la corrupción, un bajo control territorial, así como con unidades subnacionales poco funcionales, las políticas adoptadas poseen pasivos de partida para ejercer su autoridad. Así pues, aunque suene evidente, la debilidad previa del Estado será un factor central para explicar la lenta e inadecuada respuestas a desafíos. Como se explora en la siguiente sección, la segunda etapa de la respuesta estatal frente a la minería informal e ilegal, la desarticulación, evidencia este tipo de respuesta limitada por la debilidad del Estado peruano.

Esta relación entre el nivel de capacidad estatal y la respuesta efectiva/inefectiva frente a un desafío es con frecuencia correcta. No obstante, otra literatura, hoy en crecimiento, complejiza y cuestiona la relación automática entre la capacidad estatal y la respuesta del Estado. Ella puede resumirse en la idea de que hay muchas razones por las que un Estado puede actuar o dejar de actuar y puede hacerlo de distintas maneras. ${ }^{5}$ Hay determinantes políticos, económicos e internacionales que son relevantes para entender la respuesta estatal y dar cuenta de resultados diversos en términos de efectividad y tipo de respuesta en Estados con niveles de capacidad intermedia. La capacidad estatal es relevante, pero otros factores también son cruciales para entender por qué actúa el Estado, los patrones de su respuesta y la efectividad de la misma.

En esa línea, Slater y Kim (2015) muestran que a veces los Estados optan por no responder a los desafíos que se le presentan. Prefieren ignorarlos (stand-off) para no afectar directamente sus intereses al iniciar un conflicto y evitar lidiar con

$4 \quad$ No intentamos hacer una distinción absoluta entre una respuesta interna del Estado y lo que sucede fuera de este. Es obvio que el Estado se comunica con la sociedad y recoge información de ella para redefinir sus prioridades y guiar su acción. Nos referimos a que es posible distinguir cuál es el impulso primario para que la acción estatal se produzca. Consideraremos esta respuesta interna cuando el cambio en políticas y prioridades estatales emerge de los actores burocráticos y políticos ocupando cargos en el Poder Ejecutivo. Esta literatura, por supuesto, es también útil para entender las razones por las que actúan Estados desarrollados: vínculos corporativistas, intereses políticos, presiones geopolíticas e influencia de actores poderosos son todos determinantes de la respuesta estatal en cualquier Estado por fuerte que sea. 
otros problemas que pueden surgir a partir de su intervención. Podrían hacer más, pero optan por no hacerlo. Así, la inacción es producto de una decisión consciente, no una muestra de debilidad. Centrados en Estados del Sudeste asiático, los autores argumentan que el nivel de capacidad no explica del todo la decisión política de no hacer cumplir sus normas. Como veremos en el caso de estudio, la primera etapa de inacción estatal puede calzar en este patrón: varias agencias prefirieron esperar a que otras instancias se hicieran cargo del problema del oro.

En otros casos, la (in) acción estatal se explica no por falta de capacidad ni para evitar los costos de enfrentar el problema, sino fundamentalmente por las ventajas políticas que brinda no aplicar la autoridad estatal. En lo que Holland (2016) ha denominado forbearance, los políticos optan porque las burocracias a su cargo no hagan cumplir la ley a pesar de tener la capacidad para intentarlo. La prueba de ello es que, bajo otras condiciones en las que los incentivos cambian, esas mismas burocracias sí hacen cumplir la ley. Los estudios de Holland (2016) se centran en los sectores informales urbanos de América Latina, pero podemos pensar en decisiones políticas del gobierno para permitir que retadores al Estado con los que tiene vínculos de apoyo político continúen con sus actividades. Por ejemplo, como veremos, a nivel subnacional, la negativa del presidente regional de Madre de Dios para aplicar la regulación correspondiente parece responder a este patrón pues cuenta con gran apoyo electoral por parte de los mineros artesanales. La inacción más que un signo de debilidad estatal, entonces, puede ser símbolo de interés político.

Asimismo, hay casos en que una acción efectiva estatal no se da porque exista alta capacidad, sino porque actores de la sociedad colaboran con el Estado para que esta tenga lugar. En lo que denomina society driven enforcement, Amengual $(2014,2016)$ demuestra que oficinas estatales encargadas de temas ambientales y laborales con similares niveles de capacidad tienen muy distinta efectividad al hacer cumplir las normas. La respuesta para esta actuación diferenciada está en los vínculos (linkages) que tienen ciertos grupos organizados de la sociedad con estas agencias del Estado. Son estos grupos los que dotan al Estado de recursos (materiales, humanos, información, supervisión) y presionan a favor del cumplimiento de las regulaciones.

Finalmente, hay también que estudiar cómo fuentes externas al Estado pueden activar la acción estatal e incluso dar lugar a un aumento de la capacidad del Estado. Aunque observamos acciones estatales, son fuerzas externas al Estado las que llevaron a que se responda frente a un desafío. Por un lado, las presiones internacionales geopolíticas, económicas o compromisos con la comunidad internacional (a través de diversos canales como las condiciones para firmar tratados internacionales, la presión de la opinión pública transnacional, entre otras) pueden llevar a que las élites políticas creen nuevas agencias o cambien el rumbo de la política para responder a estas demandas (Dargent 2014). De forma similar, la presión doméstica también es una fuente de demanda de respuestas estatales. Actores socioeconómicos poderosos, como empresas o gremios, 
pueden reclamar respuestas estatales frente a los retadores en tanto la actividad de estos últimos afecta sus intereses. Así, las respuestas estales serán reactivas frente a la presión de estos actores.

Un aspecto interesante a evaluar es si los cambios motivados por fuerzas externas al Estado pueden dar lugar a respuestas de menor calidad, al centrarse en aspectos del fenómeno relevantes a estos actores externos. En el caso que nos ocupa la respuesta apunta a que la acción estatal privilegió aquello que las demandas externas le marcaban. Del mismo modo, resulta interesante evaluar si estas presiones pueden conducir a un cambio sustancial en la capacidad del Estado. Al motivar la acción y fortalecimiento del Estado, ¿puede este comenzar a actuar con mayor capacidad y autonomía de los intereses que motivaron su fortalecimiento? Si bien es posible, este tipo de fortalecimiento reactivo parece especialmente vulnerable a cambios externos que alteren las prioridades.

El presente caso muestra es que las presiones externas sí han llevado al fortalecimiento de las agencias estatales encargadas del control de la minería informal e ilegal a nivel nacional y subnacional. Desde la perspectiva de la capacidad entendida como posesión de recursos financieros y burocráticos que se encuentran a disposición del Estado para ejercer control sobre la sociedad y el territorio (Soifer 2008: 236), y que es usualmente aplicada en estudios de construcción de capacidad del Estado como los de Kurtz (2013), Saylor (2014) y Soifer (2015), el Estado peruano se ha fortalecido. El incremento gradual de los recursos económicos y burocráticos destinados a la prevención, detección y erradicación de la minería ilegal y la formalización de la minería informal en ambos niveles de gobierno es claro. Si bien este fortalecimiento no necesariamente ha estado acompañado de efectividad de la respuesta, el Estado es hoy más fuerte en estas áreas.

En esa línea, proponemos que en el caso se observan los siguientes determinantes de la respuesta estatal. Primero, en un inicio el Estado prefirió mirar hacia otro lado e ignorar el desafío del oro ilegal, no solo por debilidad sino también por la decisión de sus agencias de esperar que otro sector del mismo Estado se encargue del problema. Segundo, la primera respuesta estatal clara frente al fenómeno se explica por presiones internacionales que dan lugar a un cambio de prioridades en los gobiernos de la década y a la creación del MINAM. Y finalmente, el tercer gran cambio se da al sumarse a estas demandas externas una demanda doméstica de grupos mineros formales contra la minería ilegal y el desprestigio que acarreaba para su actividad. El motor de la respuesta y sus cambios fueron las presiones externas, no procesos internos al Estado.

\section{El desafío y los retadores}

Antes de analizar la respuesta estatal es preciso conocer el desafío del oro y sus varias manifestaciones. Primero, este posee una dimensión territorial, social y económica. El incremento del precio del oro ha hecho que la minería ilegal e 
informal crezca exponencialmente en diversas zonas del territorio (Defensoría del Pueblo 2014). En ellas se ha producido una migración masiva tanto a las provincias donde se localiza el mineral como a áreas vecinas (Damonte 2013), de tal manera que la explotación de oro se ha convertido en la fuente de empleo de porcentajes significativos de la población (ver cuadro 1). A la par se han desarrollado otras actividades ilegales: comercio informal, trabajo infantil, prostitución, tráfico de personas, tala ilegal, lavado de activos, contrabando, entre otros.

Cuadro 1: Principales zonas de explotación de la minería informal y población que labora en esa actividad.

\begin{tabular}{cc}
\hline $\begin{array}{c}\text { Principales zonas de explotación } \\
\text { minera informal }\end{array}$ & $\begin{array}{c}\text { Porcentaje (\%) de la PEA regional } \\
\text { provincial dedicada a la explotación } \\
\text { de minas y canteras }\end{array}$ \\
MADRE DE DIOS & 10.08 \\
Manu & 26.69 \\
Tambopata & 5.78 \\
PUNO & 3.09 \\
San Antonio de Putina & 41.58 \\
Lampa & 7.87 \\
Carabaya & 5.71 \\
\hline ICA & 1.63 \\
Nazca & 11.16 \\
AREQUIPA & 3.76 \\
Caraveli & 31.64 \\
Condesuyos & 37.23 \\
AYACUCHO & 2.44 \\
Lucanas & 10.69 \\
PUR MEDIO & 2.13 \\
\hline LA LIBERTAD & 22.23 \\
\hline
\end{tabular}

Fuente: Defensoría del Pueblo (2014) e INEI. Elaboración propia.

En segundo lugar, la minería informal e ilegal causa serios daños ambientales, especialmente en la etapa de extracción. En el caso de la minería de socavón se generan desmontes, relaves y polvo por los túneles subterráneos en los que se realiza la actividad. En la minería aluvial los efectos ambientales son más graves pues comprometen el suelo y el agua mediante la descarga de sedimentos y relaves que afectan la vida en ese ecosistema, y además dan lugar a tala 
indiscriminada en la Amazonía para el inicio de sus operaciones (Defensoría del Pueblo 2014: 183).

En tercer lugar, el desafío se vuelve mucho más complejo cuando se considera la etapa de exportación y comercialización en la cadena de valor. Hay canales formales que llevan a la salida del producto por aduanas e informales que lo sacan del país por rutas de contrabando. Generalmente se trata de empresas exportadoras legales, ${ }^{6}$ fantasmas o de fachada, que se encargan de transportar el mineral hacia refinerías en Estados Unidos o Europa a través de vía aérea y terrestre por fronteras de países vecinos con bajo control transfronterizo, como Bolivia o Ecuador (Gestión 2014).

Asimismo, hay que tener en cuenta que este es un fenómeno dinámico: el problema crece a través del tiempo. Como se verá a continuación, la demora al responder en los primeros años, cuando los retadores eran más débiles, empeoró la situación. Actualmente, existen grupos de poder vinculados al oro que se han fortalecido y que están en la capacidad de resistir los intentos de formalización por parte del Estado. En este caso, podemos distinguir entre tres tipos de grupos con intereses vertidos en la minería informal e ilegal: (i) los mineros convencionales, (ii) los procesadores y exportadores "legales", y (iii) las mafias ilegales que participan en todo el proceso. En el primer grupo se incluyen a todos aquellos trabajadores (y sus familias) que conforman la base social minera cuya subsistencia se basa en la extracción del mineral. Estos poseen un gran poder de movilización debido a su número y organización en pequeños gremios mineros. ${ }^{7}$ Por otro lado, los segundos son quienes actúan como canales formales (aunque en diversos casos con fuertes vínculos con la informalidad e ilegalidad) para procesar el oro y exportarlo legalmente. Finalmente, los terceros basan su poder en el control de circuitos de comercialización internacional y su capacidad de penetración en el aparato del Estado.

Por último, estos actores informales e ilegales han penetrado progresivamente a agencias estatales para proteger y beneficiar su actividad. La "captura" más común se produce en los organismos encargados de la fiscalización, como la policía o la fiscalía, pero también en el Poder Judicial. Incluso se llega a instancias de representación nacional y subnacional cuando se financia a candidatos políticos que pueden ser elegidos como congresistas o autoridades subnacionales (El Comercio 2014; Perú 21 2012; Los Andes 2015). Frente a este complejo reto debía reaccionar el Estado peruano.

Existe un elevado número de empresas exportadoras de oro sospechosas pues no pueden avalar la procedencia legal del mineral (La República 2015; El Comercio 2015).

Las estimaciones sobre el número de mineros informales no son exactas, pero según Torres (2015: 25), este varía entre 100.000 y 500.000 . 


\section{Las Tres Etapas de Respuesta Estatal}

En el año 2002, antes del boom, se produjo en el Perú una Ley de Formalización y Promoción de la Pequeña Minería y la Minería Artesanal y su reglamento. La norma buscaba que el marco institucional reconociera las características distintivas de la minería artesanal y pequeña minería, y brindara a los que trabajaban en ella los beneficios de la formalización. Este esfuerzo estatal se hizo para llenar los vacíos que había dejado un ordenamiento legal más interesado desde los años noventa en la gran minería y que había acabado con una serie de actividades estatales vinculadas con el fomento de la minería artesanal (Glave y Kuramoto 2005). Este marco institucional nunca fue aplicado, pues quedó desbordado por el boom.

A partir del 2004 es posible identificar tres periodos en la respuesta del Estado al fenómeno al desafío de la minería informal e ilegal de oro una vez iniciado el boom de recursos: (i) inacción (2004-2008), (ii) respuesta desarticulada (2008-2011), (iii) respuesta organizada (2011-2015). ${ }^{8}$ Los criterios centrales para la distinción de las etapas son, como se observará, la producción legal, la creación de agencias y autoridades nuevas y la cantidad de recursos asignados para combatir el retador. Estas no corresponden a cambios impulsados desde el Ejecutivo, pues ninguna de las administraciones priorizó el tema en sus planes de campaña y gobierno sino hasta que las presiones se manifestaron. En la siguiente sección explicamos qué factores motivaron el cambio en esta respuesta estatal.

\section{Primera Etapa: Inacción (2004-2008)}

Esta primera etapa se caracteriza básicamente por la inacción del Estado frente a la minería informal e ilegal. Durante este periodo, las reglas antes señaladas no tuvieron efecto; fueron letra muerta (Valencia 2014: 84). La producción legal en la materia fue escasa y la implementación de los pocos instrumentos existentes casi nula. En los primeros años de esta etapa el Estado se dedicó básicamente a realizar empadronamientos y a otorgar permisos de explotación o concesiones en diversas zonas del territorio a los mineros sin mayores requerimientos, como si la situación no hubiese cambiado radicalmente. Además de ello, en esta etapa el foco era la solución de los problemas entre las comunidades nativas amazónicas y los mineros ilegales por la invasión de terrenos para la explotación del mineral, especialmente en la región Madre de Dios. El tema aún se debatía en términos de resolución de conflictos y abolición del trabajo infantil (Valencia 2014: 85-88). No se encuentran diagnósticos estatales para resaltar la gravedad del fenómeno, ni políticas públicas diseñadas para enfrentarlo más que la vacía ley de formalización. Más aún, tal como se observa en el gráfico 
2, en ambos niveles de gobierno, los recursos destinados al control del desafío eran prácticamente nulos en esta etapa. ${ }^{9}$

Gráfico 2: Presupuesto nacional y regional destinado al control de la minería informal e ilegal

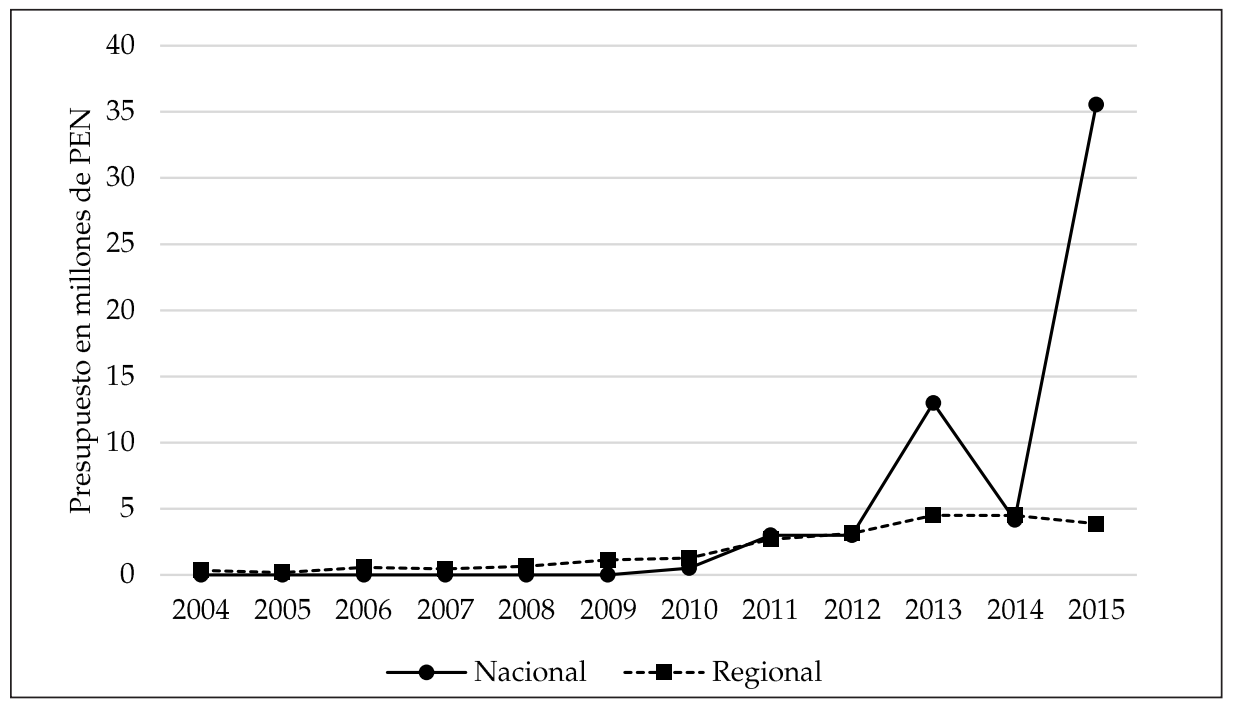

Fuente: MEF. Elaboración Propia.

Sin duda hubo mucho de debilidad estatal en este resultado, pero también de lo que Slater y Kim (2015) denominan standoffishism. El Estado no contaba con una institucionalidad adecuada, especialmente agencias estatales que se encargaran del enforcement de las reglas, pero tampoco mostró interés en enfrentar el desafío. Evidencia de ello se encuentra en la negación de responsabilidad para combatir el fenómeno tanto por parte de los gobiernos regionales como del Ministerio de Energía y Minas (MINEM) en el gobierno central. Mientras que el Estado central consideraba que el control de la minería informal e ilegal era ahora competencia de los nuevos gobiernos regionales, estos últimos, al contrario, consideraban que este era competencia del gobierno central. Por tanto, ninguna instancia quiso asumir la responsabilidad de la aplicación de la regulación. ${ }^{10}$ Además, en este periodo no se produjeron intentos para destinar más recursos, humanos y materiales, a las agencias encargadas de la formalización. Y es ilustrativa la ausencia de debate sustantivo a nivel del ejecutivo sobre la necesidad de crear un Ministerio del Ambiente y la falta de voluntad de implementar la legislación existente. Dos años después del boom, controlar la minería informal e ilegal seguían sin ser una prioridad para el Estado peruano. 
Detrás de la falta de relevancia de la problemática que ayuda a entender la inacción del Estado, se pueden identificar dos razones. Primero, como muestra el gráfico 3, a pesar de su tendencia creciente, la producción minera informal es poco significativa en términos económicos en comparación con la producción total de oro, la cual corresponde mayoritariamente a la gran minería formal y de la cual provienen las abundantes rentas para las arcas estatales. Segundo, la dimensión geográfica de la minería informal e ilegal jugó un rol importante en el patrón de respuesta del Estado en esta etapa. Ambas actividades se han desarrollado en regiones de la periferia y dentro de ellas, en provincias aún más alejadas de las capitales departamentales (la "periferia de la periferia"). Ello ayuda a entender por qué el Estado ignoró el fenómeno durante cuatro años a pesar de su crecimiento. Como se verá más adelante, solo cuando se visibilizaron los enormes daños y costos ambientales en la zona de Madre de Dios, por presiones externas, el Estado decidió actuar.

Gráfico 3: Producción minera en el Perú

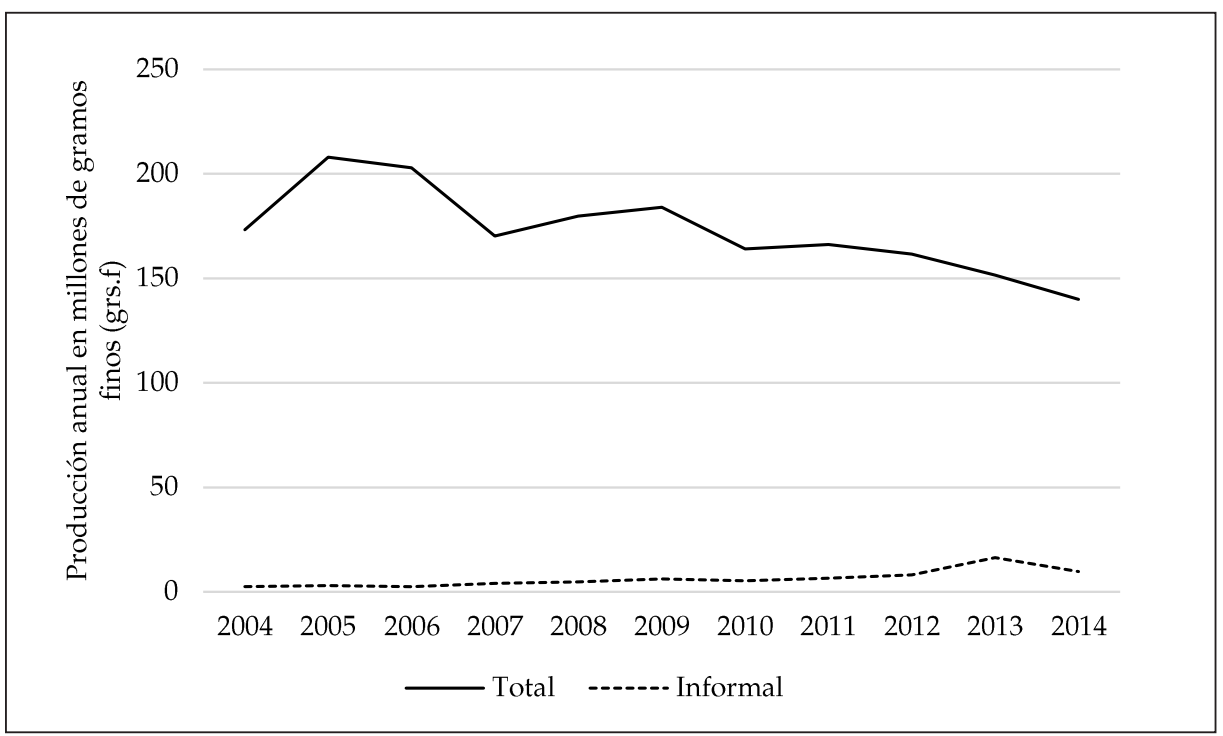

Fuente: MINEM. Elaboración propia.

\section{Segunda Etapa: Respuesta desarticulada (2008-2011)}

Esta etapa empieza con un Estado más consciente de la complejidad del problema y de haber sido desbordado por el mismo. ${ }^{11}$ Habían pasado cuatro años en los que la política de "ver y dejar hacer" del periodo anterior alimentaron 
a la minería informal e ilegal hasta volverla a una actividad mucho más visible en términos ambientales, especialmente por la destrucción del bosque y contaminación en Madre de Dios. Se puede observar entonces que el Estado reacciona formalmente, creando nuevas reglas, agencias y políticas, pero sin el enforcement de ellas y con poco cambio en la asignación de recursos. A diferencia de la etapa anterior en la que el Estado no prioriza el tema deliberadamente, en esta etapa sí se decide actuar, pero los costos de la debilidad estatal en ambos niveles de gobierno son evidentes para entender lo limitado de la respuesta.

En primer lugar, se crean algunas agencias nuevas e instrumentos de política que viabilicen la Ley de Formalización. En el 2008 se creó el MINAM al que se le asignaron competencias para regular la minería informal e ilegal dados sus peligrosos efectos ambientales, lo cual lo convertiría en uno de los actores más relevantes en la materia. Seguidamente, se produjo la modificación de la Ley de Formalización que otorgó autoridad a las Direcciones Regionales de Energía y Minas (DREM) en materia de formalización, de supervisión y sanción a lo largo de todo el proceso. Desde ese momento, estas serían las agencias encargadas directamente de la formalización. Se crean también normas de ordenamiento territorial y priorización de formalización para contener los efectos de esta minería en la destrucción del bosque, especialmente en Madre de Dios. En ese marco, en los primeros meses del 2011, y a pedido del MINAM, se empiezan a gestar respuestas represivas por parte de las Fuerzas Armadas y Policía Nacional a través de las primeras interdicciones en Madre de Dios (Valencia 2014: 134). Todo lo anterior va de la mano con un aumento de recursos destinados al control del desafío. El análisis de las leyes de presupuesto público del periodo muestra que a partir del 2009 se produce un incremento sustantivo en los recursos de los gobiernos regionales destinados a tareas de supervisión y fiscalización, y en el 2010 el gobierno central destina un pliego específico para la formalización minera. ${ }^{12}$

Una característica de la etapa es la falta de articulación entre el nivel de gobierno nacional y el subnacional. ${ }^{13}$ Con la transferencia de tareas de ejecución, fiscalización y sanción en el proceso de formalización que se otorgó a las DREM en el 2008, las oficinas competentes de nivel nacional se desligaron de las oficinas regionales sin hacerse responsables por la forma como operaban y sin coordinar con ellas las acciones que pretendían llevar a cabo. Además, las agencias nacionales no supervisaban o fiscalizaban para evaluar el cumplimiento de funciones y sancionar a las DREM de ser necesario, y tampoco invertían en el desarrollo de capacidades de estas agencias dotándolas de recursos monetarios y humanos (Defensoría del Pueblo 2014). Se les señalaban más funciones, pero básicamente con los mismos recursos. 
No obstante esta mayor actividad, en este periodo tampoco se logró mejorar el cumplimiento del marco institucional en forma sustantiva en parte por la debilidad de las áreas de fiscalización. El MINAM, que había asumido el liderazgo desde su creación, se vio desbordado por un problema de enorme complejidad que recién empezaba a comprender: la superposición de concesiones estatales en el territorio. Diversas agencias estatales habían otorgado en años previos derechos de explotación a terceros, mineros o de otro tipo, y estas concesiones no siempre eran compatibles entre sí o incluso habían cambiado de manos sin registro. El fenómeno era complicado pues su solución involucraba la acción concertada de diversas áreas del Estado, anulación o registro de una serie de títulos entregados, y el Ministerio no podía convertirse en un coordinador de todas estas actividades. Además, su estrategia había estado centrada en Madre de Dios, lo cual limitaba los alcances de las medidas en otras áreas del territorio.

Asimismo, el MINEM y el MINAM no contaban con personal especializado, lo cual llevó a que se produjeran diagnósticos incompletos del problema que se reflejan en vacíos y/o cruces legales (por ejemplo, no se contó con un protocolo que regulara de forma uniforme las interdicciones; o hasta hace pocos años, no se contaba con una estrategia que reconociera el problema incorporando la fase de exportación del oro) o en la falta de planes de acción respecto de las consecuencias sociales de la minería ilegal (Defensoría del Pueblo 2014: 207, 214). Las entidades nacionales tenían serios límites para supervisar y sancionar a los gobiernos regionales en el cumplimiento de las tareas de formalización. Por su lado, la ausencia de recursos destinada a estos sectores imposibilita, hasta la actualidad, que se establezcan más puntos de fiscalización de las vías de transporte o que se desarrollen operativos continuos de interdicción. Por lo tanto, en palabras de la Defensoría del Pueblo (2014: 22), "el marco normativo descrito anteriormente no cumplió con el objetivo de formalizar la actividad minera a pequeña escala. Los impactos sociales, ambientales, económicos de esta forma de minería se incrementaron".

\section{Tercera Etapa: Respuesta organizada (2011-2015)}

La tercera etapa se da tras la elección de Ollanta Humala en la presidencia y marca un cambio considerable en la respuesta estatal. Se trata de una etapa caracterizada por el tratamiento más integral del problema, incorporando medidas para la fase de extracción, procesamiento y exportación. En el 2014 se nombra un Alto Comisionado para la Minería Ilegal, que preside un consejo donde coordinan todas las agencias estatales involucradas en la problemática. Por otro lado, se reactiva el programa de formalización y se enfatiza una política de garrote y zanahoria.

Esta etapa muestra un mayor énfasis en la represión. El foco está en la erradicación de la minería ilegal a través de operativos de interdicción ( $\mathrm{La}$ República 2011). En esa línea, se crea la figura del Alto Comisionado que tendría 
como atribuciones establecer una Estrategia Nacional para la Interdicción de la Minería Ilegal y la elaboración de avances y resultados sobre ella. Al mismo tiempo, se pone más atención en el control de insumos químicos usados para la explotación, prohibiendo su comercialización en las zonas mineras. Asimismo, se toman medidas de coordinación e inteligencia para el control de la exportación y la venta del mineral. La policía, las Fuerzas Armadas, la SUNAT (recaudación tributaria), la SUNAD (Aduanas), el Ministerio Público y el Poder Judicial actúan concertadamente para controlar la actividad ilegal. Se comienzan a exigir a los procesadores documentos que verifiquen que el oro proviene de productores legales, por ejemplo. Además, se implementan puestos de vigilancia en las carreteras principales para supervisar la carga de los transportistas, así como se instalan dispositivos GPS en los vehículos de transporte de minerales para hacerles seguimiento (Defensoría del Pueblo 2014: 158). Como se puede observar en el gráfico 2, los picos de los años 2011 y 2013 corresponden a aumentos enormes de recursos destinados a controlar el desafío en el caso de la minería ilegal y formalización en el de la minería informal, pero también a la prevención (Defensoría del Pueblo 2014) ${ }^{14}$ Por primera vez en los presupuestos nacionales de estos años y los siguientes se destinan pliegos específicos para cada una de estas actividades. ${ }^{15}$

Sin embargo, como concluyen todos los expertos y entidades, a pesar de este fortalecimiento estatal este proceso ha sido insuficiente. La débil presencia territorial del Estado, que impide la verificación, y la complejidad de la superposición de títulos hace muy difícil ordenar una real maraña. Más aún, la concentración territorial de esta actividad en una zona de la Amazonía de difícil acceso, como Madre de Dios, incrementa enormemente los costos de llevar a cabo interdicciones. A nivel subnacional, los gobiernos regionales no cuentan con la capacidad necesaria para la política de formalización, lo que a su vez limita el éxito del conjunto. Se trata de unidades nuevas que se han visto desbordadas por las complejas funciones a desempeñar. Así, las DREM presentan escasez tanto de personal especializado como de recursos para llevar a cabo sus tareas, y además son vulnerables a la corrupción (Defensoría del Pueblo 2014: 125-129). De la incapacidad de las agencias del Estado a nivel nacional y subnacional deriva la desarticulación existente entre ambos niveles de gobierno.

Los resultados hablan por sí solos. Como se observa en el gráfico 1 presentado al inicio del artículo, a pesar de la mayor proactividad del Estado en esta etapa, la producción minera informal siguió en aumento. En términos de formalización, de las casi 60000 solicitudes de formalización presentadas por los mineros hasta fines del 2014 solo se han logrado autorizar siete (aunque incluyen varios solicitantes) en toda regla (Defensoría del Pueblo 2014: 209). Una visita de campo a Puno confirmó esta situación. Si bien Puno es la región que mejor viene trabajando el tema de acuerdo con la Defensoría, tanto actores de la sociedad 
civil como funcionarios estatales confirman los enormes problemas logísticos de la DREM. ${ }^{16}$ Asimismo, en la fiscalía ambiental de la ciudad solo se encontraron algunas denuncias por acarreo de oro, no contra mafias organizadas que son las grandes ganadoras de la actividad.

Además, estas acciones estatales han dado lugar a una respuesta social y a la adaptación de los actores ilegales. Los paros, bloqueos de carreteras, protestas masivas en las principales zonas mineras, que se extendieron a nivel nacional, son la respuesta característica a los intentos de control del Estado, tanto coercitivos como regulatorios (Perú 21 2013; La República 2014). Hay también una progresiva adaptación de los retadores para seguir exportando el oro. Así por ejemplo, un mayor control tributario da lugar a que el mineral abandone el territorio por áreas de frontera no controladas o se diseñen nuevas formas de escapar la fiscalización de aduanas y SUNAT (SPDA 2015). El Estado actúa, la sociedad se organiza y responde.

Vemos así el cambio a través de tiempo en la respuesta estatal frente a la minería informal e ilegal. Observamos que entre etapa y etapa hay variaciones importantes que han movilizado más recursos y desarrollado nuevas políticas para responder al problema. ¿Qué explica la creación del MINAM que permitió romper la inercia estatal y la conformación de una autoridad centralizada como la del Alto Comisionado, que buscó una mayor coordinación en el 2014? El propósito de la siguiente sección es explorar las causas detrás de los cambios en la respuesta estatal.

\section{EXPLICANDO LA RESPUESTA ESTATAL}

Proponemos que la respuesta del Estado ante el desafío del oro así como su evolución se explican principalmente por factores externos a este. En general, el aprendizaje del Estado ha sido bastante lento y los momentos de mayor reacción, de cambio, no se vinculan con un aprendizaje institucional interno. Como hemos adelantado, la inadecuada respuesta del Estado al inicio del fenómeno fue por una mezcla de su débil capacidad y una actitud de ignorancia deliberada. Sin embargo, las presiones externas, internacionales y domésticas, encendieron las alarmas y provocaron una respuesta y cambios. El Estado, entonces pasó de no responder a responder desarticuladamente y luego de forma más organizada.

En primer lugar, el cambio de la etapa de la inacción a la segunda etapa donde el Estado comienza a actuar ante el problema (2008-2011) puede ser explicado por la presión internacional derivada de la implementación del TLC con Estados Unidos. Para la firma del tratado se exigía como requisito previo la implementación del anexo forestal dentro del marco del capítulo ambiental. El incumplimiento de tales exigencias ponía en riesgo el tratado, un resultado 
enormemente costoso para el Estado peruano. Por ello era necesario llevar a cabo la adecuación institucional que dio lugar a la creación del MINAM en el 2008, pues el MEM no podía lidiar por sí solo con estas nuevas responsabilidades. De acuerdo con el Banco Interamericano de Desarrollo:

La creación del Ministerio del Ambiente (MINAM) mediante el Decreto Legislativo 1013, del 14 de mayo de 2008, constituye de por sí una respuesta a la necesidad interna de reorganizar la institucionalidad ambiental en el país, y asimismo constituye una reacción a la petición de Estados Unidos, en el contexto del proceso de negociación del TLC, de establecer en el país un Ministerio del Ambiente (Ruíz Muller 2011: 4).

Ahora bien, cabe señalar que si bien el énfasis del TLC se encontraba en el sector forestal y en el problema de la tala ilegal para lograr el comercio maderero sostenible, la creación del MINAM supuso que se le adjudicase competencias en materia de minería informal e ilegal pues era una actividad asociada. De hecho, la explotación artesanal de oro en zonas aluviales era la mayor actividad responsable de deforestación en la Amazonía, fundamentalmente en Madre de Dios (Gardner 2012: 307) por lo cual era imperativo desarrollar un marco institucional paralelo para abordar esta problemática. Así, las exigencias del TLC fueron el motor para crear el Ministerio y evitar la revocación del acuerdo.

Luego de su creación, el MINAM, especialmente debido a la prioridad que le dio al tema su primer ministro, el ambientalista Antonio Brack, supo aprovechar este espacio político para posicionarse como la entidad líder en la lucha contra la minería ilegal. A partir del 2010 es común ver a los ministros del Ambiente liderando operativos, presionando al gobierno central, requiriendo mayores facultades para cumplir su labor, entre otras acciones. Si bien, como se discutió antes, la labor del MINAM quedó desbordada, es en esta institución donde se aprecia un primer esfuerzo serio del Estado para responder al fenómeno. Pero un aspecto importante, y que prueba el peso de estos intereses externos al Estado, es que la acción estatal se dirigió hacia la Amazonía, zona de mayor preocupación ecológica por la tala ilegal. El grave problema del oro en otras regiones no tuvo similar atención.

En el año 2010, el tema ambiental vuelve a situarse en la mira dado que se acercaba el vencimiento del plazo del Perú para cumplir con la implementación de los acuerdos ambientales contemplados por el TLC. En ese contexto, el MINAM promulgó un Decreto de Urgencia con el objetivo de acelerar y priorizar el proceso de formalización de la minería artesanal y de pequeña escala que buscaba detener la destrucción del bosque (Damonte et al. 2013) pocos meses antes de que el plazo para implementar la agenda ambiental venciera. No obstante, no se cumplió completamente. El riesgo de sanción era bastante real y el malestar de los funcionarios estadounidenses se manifestó en los medios de comunicación, pues estos ejercieron presión durante los siguientes meses para que el Estado atienda el problema de la protección de los bosques y 
cumpla el anexo forestal adecuadamente (Perú 21 2010; Info Región 2010a, 2010b; Europapress 2011).

Como se señaló en apartados anteriores, al tomar acciones se descubre la complejidad del problema y la enorme dificultad de formalizar la minería informal y de reprimir a la ilegal. Las actividades de interdicción son muy costosas, requieren ingentes recursos para el desplazamiento territorial y continuidad en el tiempo. Este primer impulso no logra ser efectivo, pues el MINAM estaba lejos de poder coordinar a todas las entidades a cargo del control de la minería ilegal y de superar el problema de las concesiones.

Es en este contexto que una nueva forma de presión internacional al Estado se manifiesta y en conjunto con la anterior conducirán a la tercera etapa (2011-2015). Grupos ambientalistas transnacionales mediatizan la urgencia de actuar en el caso de Madre de Dios. A partir del año 2011, una serie de estudios académicos internacionales muestran el enorme y rápido impacto ambiental de la minería en la región, fundamentalmente en materia de desforestación, daños a la salud por el uso inadecuado y elevado de mercurio, y perjuicios a los ecosistemas. Algunos de estos reportes son los de Swenson et al. (2011), Gardner (2012) y Amazon Conservation Association (2014). La imagen que se proyecta es de una actividad destructiva que crece sin control y de un Estado débil que carece del interés para enfrentarla. La prensa local recoge estas denuncias.

Un segundo factor adicional externo al Estado es la presión doméstica por parte de actores socioeconómicos con poder que se ven afectados por la minería informal e ilegal o consideran se les imponen estándares ambientales mientras que los "reales" contaminadores no son regulados. Los principales fueron la Sociedad Nacional de Minería, Petróleo y Energía (SNMPE) y la Confederación Nacional de Instituciones Empresariales Privadas (CONFIEP). Se trata de sectores vinculados con mineros formales que pertenecen a escalas mayores de actividad, especialmente los medianos que compiten con los informales e ilegales por su territorio, y grandes que consideran perjudicada su imagen por este tipo de minería y pueden resaltar los beneficios de la formalidad. La Comisionada Alicia Abanto de la Defensoría del Pueblo señala que este grupo realiza desde hace años demandas para que se reconozcan los costos de la minería informal (Entrevista a Abanto 2014).

La presión visible de estos grupos de mineros formales se hizo sentir a través de los medios de comunicación, en los que publicaron comunicados institucionales y organizaron una serie de eventos exigiendo la actuación decidida del Estado para enfrentar el fenómeno. Así, desde el 2010 los líderes de los gremios empresariales mineros comenzaron a advertir sobre la asociación de la minería ilegal e informal con el narcotráfico y el lavado de activos, la movilización de grandes capitales sin el pago de impuestos ni regalías, la existencia de mafias, así como a demandar acciones estales estrictas no reversibles y felicitar los avances del gobierno en la materia, siempre buscando dejar en claro su posición de actividad formal no destructiva diferente a la informal e ilegal (El Comercio 
2010). Así podemos ver una serie de comunicados demandando una respuesta. ${ }^{17}$ Estas comunicaciones tuvieron gran resonancia en los medios locales y marcaron en muchos casos la agenda de debate en torno a la minería informal e ilegal. ${ }^{18}$ Tales presiones y la visibilización de la problemática alcanzada como resultado obligaron al Estado a actuar.

Con el cambio de gobierno en el 2011, entonces, se da mayor prioridad a la lucha contra la minería formal e ilegal. En esta tercera etapa se retoman las acciones represivas de interdicción a través de operativos más ambiciosos, pero también existe más voluntad de diálogo con las organizaciones mineras, con los afectados por la superposición de autorizaciones (agricultores, comunidades nativas) y con los gobiernos regionales, los cuales, a su vez, muestran mayor disposición para colaborar con el Ejecutivo. El cambio sorprendió, pues Humala había mostrado en su campaña cercanía con las asociaciones de mineros informales y gozaba del apoyo de varios dirigentes. Se pensó que su gobierno tendría mejores relaciones con la minería informal, tal vez si no de apoyo directo si de permisividad. Consideramos que las presiones antes señaladas llevan a este cambio de prioridades: presiones internacionales, compromisos externos, grupos de interés y una creciente demanda doméstica para responder al fenómeno.

Salomón Lerner, primer Presidente del Consejo de Ministros del gobierno de Ollanta Humala, añade a esta explicación la preocupación del Presidente por el tema de la seguridad y la criminalidad vinculada a la minería ilegal. Los estudios sobre los graves impactos de la minería informal e ilegal en la Amazonía, así como informes sobre las vinculaciones de esta actividad con una serie de actividades delictivas y del narcotráfico influyeron en la prioridad del tema en la agenda del nuevo gobierno (Entrevista a Lerner, 2014). Lamentablemente no contamos con información suficiente para determinar si estas presiones de seguridad fueron también internacionales o si, más bien, mostrarían que otras áreas del Estado vinculadas con la seguridad influyeron sobre el Presidente.

\section{CONCLUSIÓN}

El caso analizado permite concluir que la respuesta limitada del Estado ante el desafío de la minería informal e ilegal así como su evolución a lo largo del tiempo se explica principalmente por causas externas, como la influencia del

17 SNMP: Minería ilegal en Perú mueve más de US\$ 900 millones que no pagan impuestos y regalías (22 de febrero de 2011); CONFIEP: Narcotráfico “invierte" US\$ 500 millones al año en minería ilegal (14 de febrero de 2012); SNMPE: Destaca voluntad política del gobierno para erradicar la minería ilegal en Perú (20 de febrero de 2012); SNMPE: Gobierno no se enfrenta a minería de subsistencia sino a mafias que financian extracción ilegal de minerales (14 de marzo de 2012); SNMPE: ni un paso atrás en la lucha contra la minería ilegal (16 de mayo de 2012); SNMPE: Minería formal y moderna se diferencia claramente de la extracción ilegal de minerales (14 de mayo de 2012).

18 Al parecer, este pedido también habría sido directo. Si bien no tenemos verificada dicha información, un informante en condición de anonimato nos comentó que en el marco de las negociaciones de un nuevo impuesto a las mineras al inicio del gobierno los mineros medianos demandaron al gobierno una mejor respuesta frente a la minería ilegal. 
entorno social doméstico e internacional. En general, el aprendizaje del Estado ha sido bastante lento y los momentos de adaptación se vinculan a estos factores, más que a un cambio interno. Solo en la tercera etapa vemos un Estado más proactivo, con una respuesta más integral y más capacidad de agencia, demostrando que, dentro de su debilidad, sí hay mejores respuestas posibles.

Consideramos que este tipo de respuesta reactiva deja dudas como mecanismo de fortalecimiento estatal sostenible. Al estar determinada por los intereses externos al Estado (ambiental y empresarial) se enfoca en ciertos temas relevantes para estos actores externos antes que por una evaluación más global del problema. En la segunda etapa, por ejemplo, la agenda del MINAM estuvo más dirigida al pasivo ambiental de Madre de Dios, el caso más urgente y visible, que hacia otras muchas áreas donde la minería informal se sigue desarrollando y de forma más intensa que en el pasado. De forma similar, en la tercera etapa puede apreciarse cómo el Estado termina privilegiando acciones simbólicas de erradicación, impactantes, pero que no siempre son las más efectivas para controlar el problema. Entonces, sin actores estatales con capacidad de mantener su agenda, el cambio en estas presiones externas y la pérdida de relevancia del tema en la agenda pública puede hacer perder interés en combatir el fenómeno.

Sin embargo, una pregunta abierta es si este fortalecimiento institucional, aunque limitado, sea mayor de lo observado hasta el momento. Si el precio del mineral cae y los retadores pierden poder, se podrá observar si las reglas, organizaciones y experiencia ganada por el Estado marcan una mejor regulación en tiempos normales y una mayor capacidad de agencia para impulsar sus propias políticas. En forma más general, tomar conciencia de las distintas maneras en que fuerzas sociales o grupos de interés pueden empujar la acción estatal y su fortalecimiento abre una agenda interesante de investigación sobre las fuentes de capacidad estatal, especialmente de agencias que destacan por su mayor capacidad en entornos estatales débiles.

El presente trabajo también presenta ideas e información relevante para estudios comparados de economía política que exploren el impacto local y los legados del boom de recursos. El mismo factor externo, en este caso el aumento del precio del oro, puede tener diversas manifestaciones locales dependiendo de factores diversos discutido en este trabajo, como la fortaleza previa de instituciones y organizaciones estatales, el poder de grupos sociales y criminales fortalecidos por el boom, el tipo de gobierno en el poder, o las economías ilegales existentes antes del boom. A diferencia del Perú, ¿el marco institucional para la pequeña minería y la capacidad de agencias estatales vinculadas a esta actividad que existían antes del boom permitieron canalizar mejor el fenómeno en países vecinos como Bolivia, Colombia y Ecuador? ¿O en todos ellos las instituciones y el Estado fueron desbordadas? ¿Qué efectos tendrá que se apropien de las rentas del oro grupos armados o mafias regionales como en Colombia? ¿Y si los beneficiados son grupos sociales, como las cooperativas auríferas en Bolivia, que por sus vínculos políticos reciben un trato tolerante por parte del Estado? Todas 
preguntas abiertas vinculadas al oro, pero que fácilmente pueden asociarse a otros fenómenos políticos vinculados al boom de recursos.

\section{REFERENCIAS}

\section{Fuentes oficiales}

CONFIEP. 2012. Narcotráfico "invierte" US\$ 500 millones al año en minería ilegal (14 de febrero)

SNMP. 2011. Minería ilegal en Perú mueve más de US\$ 900 millones que no pagan impuestos y regalías (22 de febrero).

SNMPE. 2012. Destaca voluntad política del gobierno para erradicar la minería ilegal en Perú (20 de febrero).

SNMPE. 2012. Gobierno no se enfrenta a minería de subsistencia sino a mafias que financian extracción ilegal de minerales (14 de marzo).

SNMPE. 2012. Minería formal y moderna se diferencia claramente de la extracción ilegal de minerales (14 de mayo).

SNMPE. 2012. Ni un paso atrás en la lucha contra la minería ilegal (16 de mayo).

\section{Prensa}

Caretas. 2010. "Alerta Roja Forestal". Caretas (6 de agosto).

El Comercio. 2010. "Confiep: Minería ilegal estaría ligada a lavado de dinero y narcotráfico". El Comercio (6 de abril).

El Comercio. 2014. "Congresista 'Comeoro' se reúne con mineros de Chala y Nasca”. El Comercio (1 de marzo).

El Comercio. 2015. "Red de 'Peter Ferrari' exportó 14 toneladas de oro a EE.UU". El Comercio (22 de marzo).

Europapress. 2011. "Perú y EEUU revisarán cumplimiento del anexo forestal del TLC en Washington". Europapress (24 de abril).

Gestión. 2014. "Lucha contra minería ilegal de oro en Perú crea ruta de contrabando por Bolivia". Gestión (25 de noviembre).

Info Región. 2010a. "Estados Unidos denunció que Perú no implementó al 100\% addenda forestal del TLC". Info Región (3 de abril).

Info Región. 2010b. "Del TLC al TFallé, una vergüenza internacional". Info Región (5 de agosto de 2010).

La República. 2011. "Estado golpea a minería informal. 'Aurum I' desplaza 1.500 efectivos de las FFAA y PNP en operativo combinado". La República (6 de noviembre).

La República. 2014. "Mineros de cinco regiones llegan a acuerdo con PCM y levantan huelga". La República (26 de marzo).

La República. 2015. "Peter Ferrari exportó oro de minería ilegal por 637 millones de dólares". La República (22 de marzo).

Los Andes. 2015. “Un grupo de mineros artesanales aspiran formar agrupación política nacional". Los Andes (17 de julio).

Perú 21. 2010. "EE.UU. denuncia incumplimiento de Perú", Perú 21(30 de julio).

Perú 21. 2012. "Gobernador de Madre de Dios apoya a la minería ilegal". Perú 21 (31 de diciembre).

Perú 21. 2013."Perú: Mineros informales acatan paro por segundo día consecutivo". Perú 21 (1 de octubre). 


\section{Entrevistas}

Abanto, Alicia. Comisionada de la Defensoría del Pueblo. Lima, 15 de enero del 2014.

Choquehaunca, Zenón. Director de Asociación Servicios Educativos Rurales, Sede Puno. Puno, 12 de marzo 2015.

Galliani, Luis. Empresario formal de procesamiento de oro. Lima, 15 de febrero de 2014.

Herrera, Alessandra. Funcionaria del Ministerio de Energía y Minas. Lima, 23 de enero del 2014.

Lerner, Salomón. Ex Presidente del Consejo de Ministros (2011). Lima, 25 de febrero 2015.

Raez, Ernesto. Asesor del Ministerio del Ambiente. Lima, 31 de enero del 2014.

Salas, Dante. Director de la Dirección Regional de Energía y Minas, Puno. Puno, 12 marzo de 2015.

\section{REFERENCIAS BIBLIOGRÁFICAS}

Alvarado Barbarán, Laura. 2014. "Impactos y lecciones aprendidas de la minería ilegal e informal en Madre de Dios". Economía y Sociedad 84(1): 56-63.

Amazon Conservation Association. 2014. Fact Sheet: Illegal Gold Mining in Madre de Dios, Peru. Consulta 25 de febrero de 2015, http:/ / www.amazonconservation.org/pdf/gold_mining_fact_sheet.pdf

Amengual, Mathew. 2016. Politicized Enforcement in Argentina. New York: Cambridge University Press.

Amengual, Matthew. 2014. "Pathways to Enforcement: Labor Inspectors Leveraging Linkages with Society in Argentina". Industrial and Labor Relations Review 67(1): 3-33.

Ashe, Katy. 2012. "Elevated Mercury Concentrations in Humans of Madre de Dios, Peru". PLOS ONE 7(3): 1-6.

Damonte, Gerardo. 2013. "Formalizing the Unknown. The Stalemate over Formalizing Small-scale Mining in Madre de Dios". The Broker. Consulta 23 de febrero de 2015, http:/ / www.thebrokeronline.eu/Articles/Formalizing-the-unknown

Damonte, Gerardo; Bueno de Mesquita, Mourick; Pachas, Víctor Hugo y Mary Chávez. 2013. "Small-scale Gold Mining and Social and Environmental Conflict in the Peruvian Amazon". En Small-scale Gold Mining in the Amazon: The Case of Bolivia, Brazil, Colombia, Peru and Suriname, editado por Leontien Cremers, Judith Kolen y Marjo de Theije. Amsterdam: CEDLA, 68-84.

Dargent, Eduardo. 2014. "Determinantes internacionales de la capacidad de las agencias estatales". Apuntes 41 (74): 9-40.

Defensoría del Pueblo. 2014. Gestión del Estado frente de la minería informal e ilegal en el Perú. Informe $\mathrm{N}^{\circ}$ 167. Lima: DP.

Eaton, Kent. 2012. "The State of the State in Latin America: Challenges, Challengers, Responses and Deficits". Revista Ciencia Politica 32(3): 643-657.

Gardner, Elie. 2012. "Peru Battles the Golden Curse of Madre de Dios". Nature 486: 306-307.

Giraudy, Agustina. 2012. "Conceptualizing State Strength: Moving Beyond Strong and Weak States". Revista de Ciencia Política 32(3): 599-611.

Glave, Manuel y Juana Kuramoto. 2007. “La minería peruana: lo que sabemos y lo que aún nos falta por saber". En GRADE. Investigación, políticas y desarrollo en el Perú. Lima: GRADE, 135-181.

Holland, Alisha. 2016. "Forbearance". American Political Science Review 110(2): 232-246.

Kurtz, Marcus. 2013. Latin American State Building in Comparative Perspective: Social Foundations of Institutional Order. New York: Cambridge University Press.

Moschella Miloslavich, Paola. 2011. Impactos ambientales de la minería aurífera y percepción local en la microcuenca Huacamayo, Madre de Dios. Tesis de Licenciatura. Pontificia Universidad Católica del Perú.

Mosquera, César. 2009. Estudio diagnóstico de la actividad minera artesanal en Madre de Dios. Lima: Fundación Conservación Internacional. 
Ruíz Muller, Manuel. 2011. “Análisis del proceso de implementación del capítulo ambiental del acuerdo de promoción comercial entre Estados Unidos y Perú: La situación peruana". Resumen de Políticas BID-RP-127: 1-52.

Organismo de Evaluación y Fiscalización Ambiental. 2013. Fiscalización ambiental a la pequeña minería y minería artesanal. Lima: OEFA.

Orihuela, José Carlos. 2014. "Bonanza minera, conflictos y salidas institucionales a nivel lo-

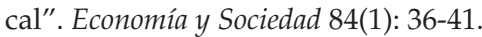

Pachas, Víctor Hugo. 2013. Conflictos sociales en Madre de Dios: El caso de la minería en pequeña escala de oro y la ilegalidad. Lima: CEAS.

Paredes, Maritza. 2013. Shaping State Capacity. A Comparative Historical Analysis of Mining Dependence in the Andes, 1840s-1920s. Tesis doctoral. Universidad de Oxford.

Ruíz, Manuel. 2011. "Análisis del proceso de implementación del capítulo ambiental del acuerdo de promoción comercial entre Estados Unidos y Perú. La situación Peruana". Resumen de Políticas. Lima: BID.

Saylor, Ryan. 2014. State Building in Boom Times: Commodities and Coalitions in Latin America and Africa, New York: Oxford University Press.

Slater, Dan y Diana Kim. 2015. "Standoffish States: Nonliterate Leviathans in Southeast Asia". Trans-Regional and National Studies of Southeast Asia 3(1): 1-20.

Sociedad Peruana de Derecho Ambiental. 2014. La realidad de la minería ilegal en países amazónicos. Lima: SPDA.

Sociedad Peruana de Derecho Ambiental. 2015. Las rutas del oro ilegal: Estudios de caso en cinco países amazónicos. Lima: SPDA.

Soifer, Hillel. 2008. "State Infrastructural Power: Approaches to Conceptualization and Measurement". Studies in Comparative International Development 43(X): 231-251.

Soifer, Hillel. 2015. State Building in Latin America. New York: Cambridge University Press.

Stein, Ernesto y Mariano Tomassi. 2007. "The Institutional Determinants of State Capabilities in Latin America". En Annual World Conference on Development Economics Regional: Beyond Transition, Francois Bourguignon y Boris Plescovik (editores). Washington: BID, 193-225.

Swenson, Jennifer, Catherine Carter, Jean-Christophe Domec y Cesar Delgado. 2011. "Gold Mining in the Peruvian Amazon: Global Prices, Deforestation, and Mercury Imports". PLOS ONE 6(4): 1-7.

Torres, Víctor. 2015. Minería informal e ilegal en el Perú: Impacto socioeconómico. Lima: CooperAcción.

Valencia, Lenin. 2014. Madre de dios: ¿Podemos evitar la tragedia? Políticas de ordenamiento de la minería aurífera. Lima: SPDA.

Eduardo Dargent es PhD en Ciencia Política por la Universidad de Texas en Austin y MA en filosofía política por la Universidad de York. Es profesor del departamento de Ciencias Sociales de la Pontificia Universidad Católica del Perú, director del programa de maestría de la Escuela de Gobierno y Políticas públicas de la misma casa de estudios, así como coordinador del Grupo de Investigación sobre Capacidad del Estado peruano (GICEP) de la PUCP. Ha publicado los libros Technocracy and Democracy in Latin America: The Experts Running Government (Cambridge University Press, 2015) y Demócratas Precarios (IEP 2009).

Madai Urteaga es estudiante de pregrado de Ciencia Política y Gobierno de la Pontificia Universidad Católica del Perú (PUCP). Actualmente es asistente de investigación en la Pontificia Universidad Católica del Perú y miembro del Grupo de Investigación sobre Capacidad del Estado Peruano (GICEP). 
